\title{
AC Loss Comparison between Multi-filament and Non-striated YBCO Coils Designed for HTS Propulsion Motors
}

\author{
Dong Xing, Jay Patel, Qixing Sun, Jiahui Zhu, Guomin Zhang, Fei Liang, Weijia Yuan and Min Zhang
}

\begin{abstract}
In this paper, the properties of current capacity and $\mathrm{AC}$ transport loss of striated HTS coil is compared with ordinary HTS coil by using both experimental and simulation methods. The measurements were carried out by transporting a sinusoidal varying current at $77 \mathrm{~K}$, with an amplitude range of $10-50 \mathrm{~A}$ in frequency from $70 \mathrm{~Hz}$ up to $300 \mathrm{~Hz}$. Measurement facilities and methods are explained in more detail in the paper. The critical current of the $4 \mathrm{~mm}$ width multi-filament coil made with 4 filaments at a spacing $0.03 \mathrm{~mm}$ was found to be lower than that of the non-striated coil. The frequency dependent characteristics agreed well in both experimental and simulated results. Reducing AC loss of HTS is one enabling factor for widespread adoption of the technology and therefore understanding its characteristics is important and discussed in this paper. Future plans based on this preliminary work is the testing of multi-filament tapes in an axial flux motor field environment.
\end{abstract}

Index Terms - AC loss, Superconducting coils, Multi-filament superconductors, Loss measurement.

\section{INTRODUCTION}

YBCO coated conductors are regarded as one of the most promising coated conductors due to their high current density and ability to maintain high critical current within strong magnetic fields [1]. Of the handful of barriers preventing widespread adoption of HTS technology is the problem of AC losses. Indeed, many applications of superconductors that allow them to integrate into live systems require alternating current to be carried i.e. motors, generators or transformers. $\mathrm{AC}$ losses and remnant fields can be reduced by using multifilament tapes, as found by Brandt, the magnetization loss follows a square law with the width of the superconductor [2]. Multi-filament tape is striated using a variety of techniques, the most popular methods are laser scribing or chemical etching. The improvement of manufacturing techniques have allowed the use of narrower filaments and results have proved that the thinner the individual filaments, the lower the undesirable AC losses. Additionally, striated HTS can also

Dong Xing Jay Patel, Qixing Sun, Fei Liang, Min Zhang, Weijia Yuan are with the Department of Electronic and Electrical Engineering, University of Bath, Bath BA2 7AY, United Kingdom. (E-mail: dx278@bath.ac.uk).

J. Zhu is with the China Electric Power Research Institute, Beijing 100192, China. She is also with the Department of Electronic and Electrical Engineering, the University of Bath, Bath BA2 7AY, U.K.

Guomin Zhang is with Chinese Academy of Science, Beijing. reduce current inhomogeneity caused by screening currents, which are crucial to applications such as NMR and MRI.

A great number of research groups as well as manufacturers have been focusing on investigating the magnetization $\mathrm{AC}$ loss and transverse resistivity of striated tapes [3-8]. Lots of researchers has confirmed that striation is an effective way to reduce the hysteresis AC losses. Meanwhile, the coupling loss becomes more important with an increase in the number of filaments. The superconductor can experience full filament uncoupling to full coupling within a specific frequency range [4]. Majoros et al. gave more explanations on the screening current coupled in both striated and monolayer samples [9]. Although lots of results have been gained for straight tape 'infield' scenarios, the transport AC loss of YBCO CC coils is an aspect which cannot be ignored with applied current. Majoros et al. compared the transport AC loss between striated (multifilament) and non-striated YBCO CC tape and addressed that the frequency dependence for transport loss is rather weak and mainly determined by the losses in the substrate and Ni layer up to currents of about $50 \%$ of the critical current [10]. Finite element methods are an effective way to analyze the critical state and characteristics of the coil. F. Grilli et al. [11], Z. Hong et al. [12], E. Pardo et al. [13], Souc et al. [14] and M. Zhang et al. [15] have promoted the numerical scheme for AC loss calculation to more efficient levels. M. Zhang et al. has used an experimentally verified H-Formulation to study air cored and distributed windings in motors [16]. However, the transport AC loss of multi-filament YBCO coils have not been discussed clearly in both experiment and simulation parts. Therefore, we will investigate the properties of transport AC loss of multi-filament and non-striated coils by using both experimental and finite element method of an axial symmetrical model combining the methods presented by $\mathrm{Z}$. Hong et al. in [12] and $\mathrm{M}$ Zhang et al. in [15].

In this paper, we present the critical current measurement as well as the $\mathrm{AC}$ transport loss measurement of two coils wounded by multi-filament YBCO CC and normal nonstriated YBCO CC manufactured by Superpower. The width and thickness of both of the tapes are same. Critical currents and the transport $\mathrm{AC}$ losses are measured using the four-point and compensation method. Experimental results are also compared with simulated results. The coils considered are intended to be used in a prototype axial flux motor; a model for considering the $\mathrm{AC}$ loss by coupling a machine model with a HTS model was proposed by M. Zhang et al. [17] and will be used to verify the machine results in high-frequency propulsion situation. 


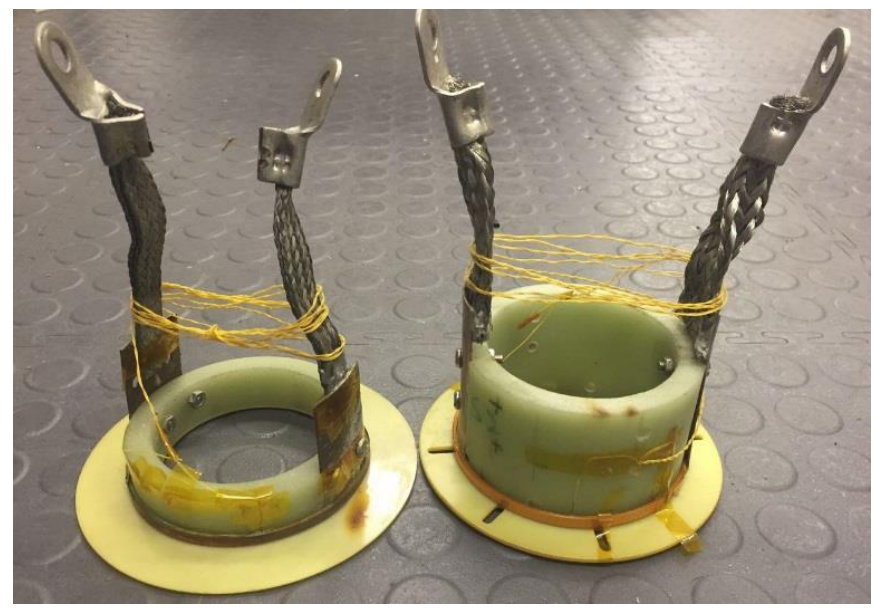

Fig. 1. Multi-filament sample coil (left) and non-striated sample coil (right) wounded by YBCO coated conductors.

\section{EXPERIMENT}

\section{A. Sample Preparation}

Two sample coils wound using YBCO coated conductors were manufactured and are shown in figure 1 . The tapes used are supplied by Superpower. The width and the length of the two striated and non-striated tapes are identical with a width and length of $4 \mathrm{~mm}$ and $2.76 \mathrm{~m}$ respectively. The tapes consist of $50 \mu \mathrm{m}$ hastelloy substrate and $2 \mu \mathrm{m}$ silver protective layer. The Kapton ${ }^{\circledR}$ film thickness is $0.03 \mathrm{~mm}$, total tape thickness with adhesives is $0.10 \mathrm{~mm}$ with $0.04 \mathrm{~mm}$ adhesive on each side. The multi-filament tape has 4 filaments with a $0.03 \mathrm{~mm}$ gap per filament. The measurements were carried out using two sample holders which have outer and inner diameters of $8.9 \mathrm{~cm}$ and $7 \mathrm{~cm}$. Both of the coils are wound with 9.5 turns. More details are listed in Table 1.

TABLE I

\begin{tabular}{lcc}
\hline \hline & & \\
& Striated coil & Non-striated coil \\
\hline Wire Type & SCS4050-AP & SCS4050-AP-i \\
Tape width & $4 \mathrm{~mm}$ & $4 \mathrm{~mm}$ \\
Total tape length $(\mathrm{m})$ & 2.76 & 2.76 \\
Number of filaments & 4 & - \\
Filament width(mm) & 0.978 & - \\
Tape Ic (A) & 90 & 103 \\
Number of Turns & 9.5 & 9.5 \\
Coil radius (mm) & $44.5 \mathrm{~mm}$ & $44.5 \mathrm{~mm}$ \\
$\begin{array}{l}\text { Frequency range } \\
\text { (Hz) }\end{array}$ & $70-300$ & $70-300$ \\
Coil Ic (A) & 66 & 71 \\
\hline
\end{tabular}

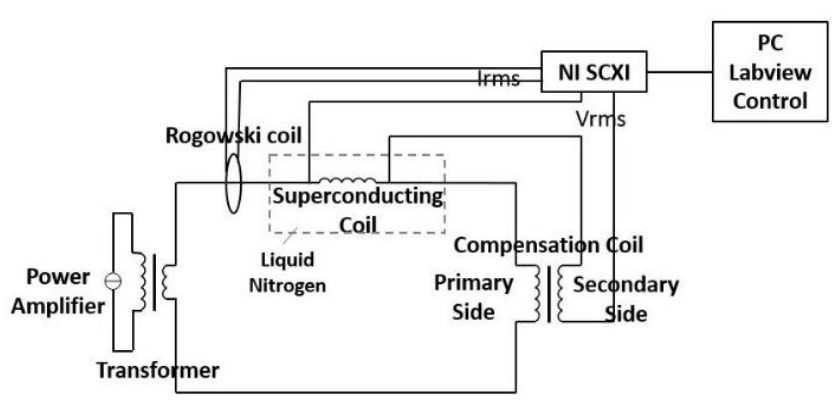

Fig. 2. Measurement scheme of the transport AC loss measurement

\section{B. Critical Current Measurements}

The critical current of the coils is measured by the fourpoint method using a $1 \mu \mathrm{V} / \mathrm{cm}$ criterion in self-field conditions in the liquid nitrogen at $77 \mathrm{~K}$ operating temperature. The ramp rate of the applied current can be controlled with LabVIEW and NI data acquisition module at $1 \mathrm{~A} / \mathrm{s}$. A LabVIEW interface panel is used to control the DC power supply. The maximum voltage through the HTS sample is set as 10 times the critical voltage.

\section{AC Transport Loss Measurements}

The AC loss testing system we used in this measurement is based on the experiment system established and explained by Z. Jiahui et al.[18]. The AC loss measurement system panel is shown in Fig. 2. A sinusoidal signal waveform is supplied by a $20 \mathrm{~kW}$ power amplifier which is connected to a transformer. The Rogowski coil is used to measure the applied current from the secondary side of the transformer. To get a pure resistive voltage, a cancellation coil has been connected in series with the measured superconducting coil and the secondary side of the transformer to cancel the inductive voltage produced due to a time varying applied transport current. The self-field AC transport loss of the superconducting coil per unit length per cycle can be calculated by equation 1 :

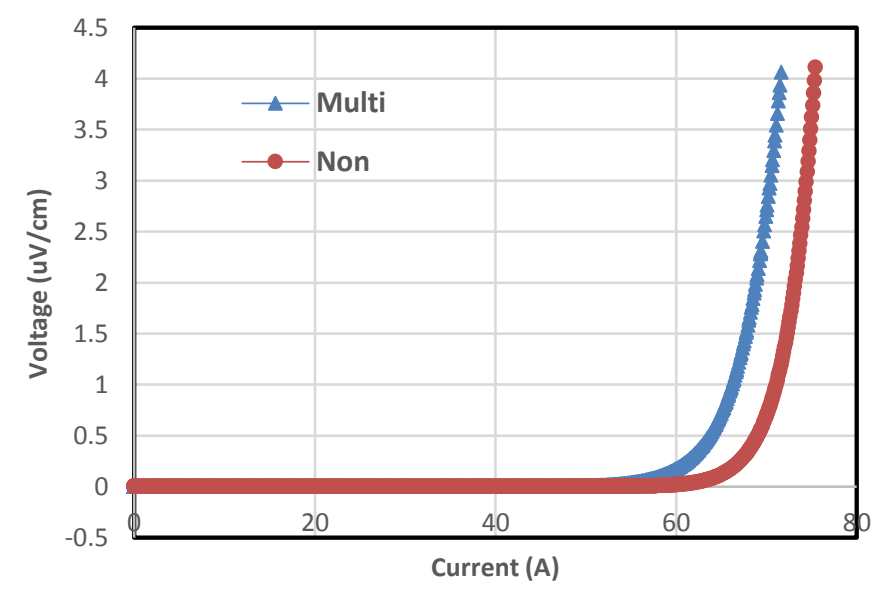

Fig. 3. Critical current of multi-filament coil (left line) and non-striated coil (right line) after processed in MATLAB. 


$$
Q=\frac{V_{r m s} I_{r m s}}{f L}
$$

where Vrms and Irms are RMS values of pure resistive loss voltage and the AC transport current of the superconductor is in phase with Vrms between the two voltage taps. ' $f$ ' is the frequency. ' $L$ ' is the length between two voltage taps. The range of Irms is varied from 10 to $50 \mathrm{~A}$ owing to the limits of the power amplifier supply. The in-phase voltage and current can be observed from the Labview software. When the resistive voltage is cancelled well by the cancellation coil, the sinusoidal voltage and current line will coincide. There are five frequencies chosen: 70,120,170,220 and $300 \mathrm{~Hz}$.

\section{RESULTS}

\section{A. Critical Current}

Figure 3 indicates the DC current and voltage characteristics for striated and non-striated coils, the coil with no strips has the higher critical current which is around $71 \mathrm{~A}$. Due to the removal of superconducting material, the critical current of the multi-filament coil is $66 \mathrm{~A}$ which is about a $7 \%$ degradation of the non-striated coil. In regards to the filament

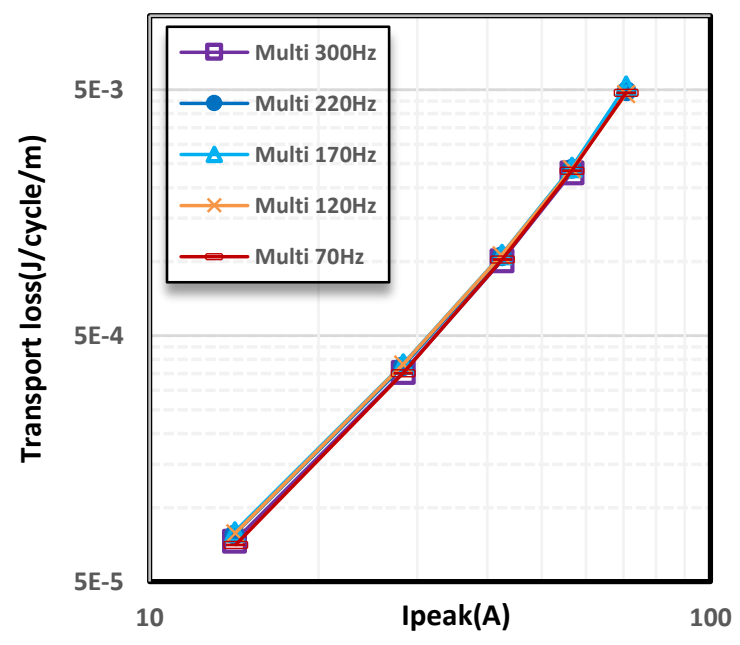

(a)

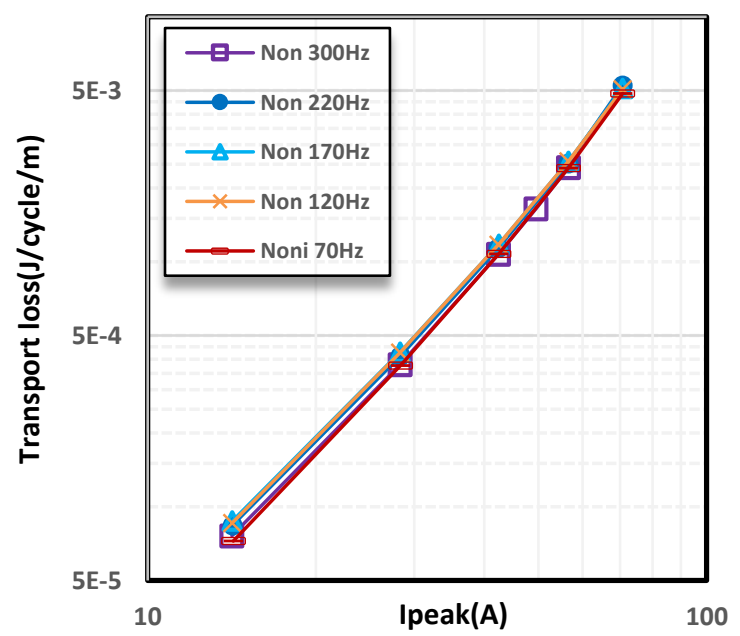

Fig. 4. Transport AC loss of (a) Multi-filament and (b) non-striated coils with various applied currents measured in the frequency range $70 \mathrm{~Hz}$, to $300 \mathrm{~Hz}$ and peak current range from $14 \mathrm{~A}$ to $70 \mathrm{~A}$. width, the cut groove is quite thin $(0.03 \mathrm{~mm})$, whereas the critical current still decreases a lot for a 9.5-turn coil. The $\mathrm{n}$ values are calculated by MATLAB, which are 18 and 24 for the multi-filament and non-striated coils respectively.

\section{B. AC Losses}

The experimental measurement results of transport AC loss of multi-filament and non-striated coils are shown in figure 4. Both of them are applied with the same current and in the same frequency range $70 \mathrm{~Hz}$ to $300 \mathrm{~Hz}$. The rms current range for most of the tests is from $10 \mathrm{~A}$ to $50 \mathrm{~A}$. The peak current range for most of the tests is from $14 A$ to $71 A$.The rms current cannot reach $50 A$ for higher frequency, due to the upper limits of the internal oscillator output of the power amplifier in our lab. Compared with the multi-filament coil, a larger difference can be witnessed from figure 4(b) where the AC losses of the non-striated coil at $170 \mathrm{~Hz}$ is higher than the AC losses at $70 \mathrm{~Hz}$ with lower transporting current. It indicates that at lower currents, the AC losses of the multi-filament coil are more independent of frequency than the non-striated coil.

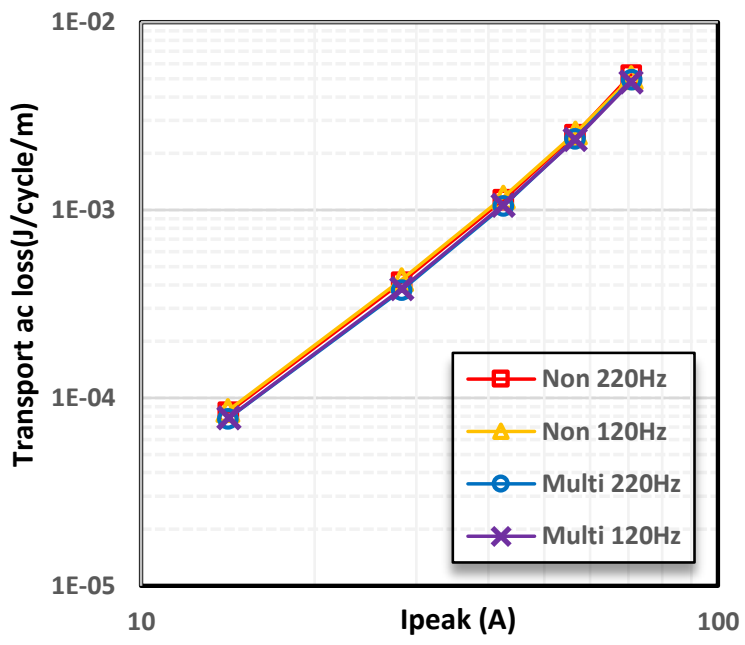

(a)

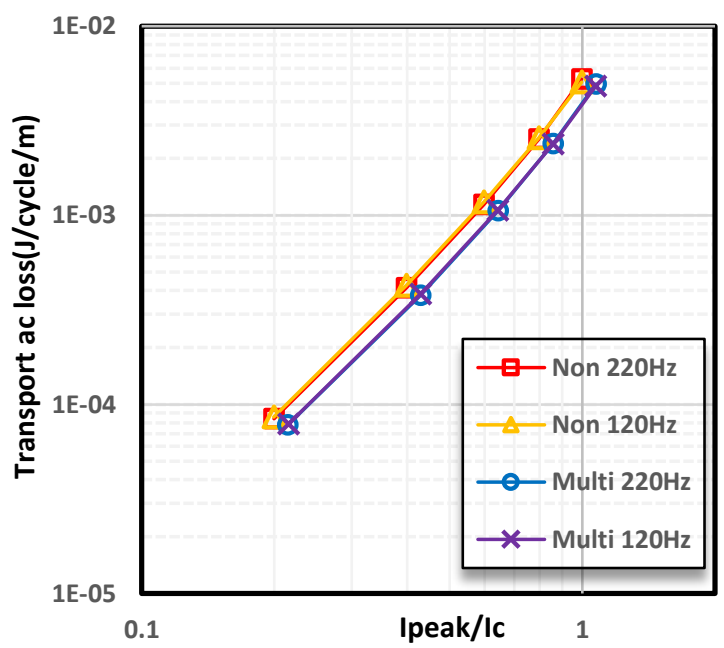

Fig. 5. Comparison of the transport AC loss with (a) applied current and (b) normalized current of multifilamentary and non-striated coils derived from experiment in the frequency range $70 \mathrm{~Hz}$ to $300 \mathrm{~Hz}$. 


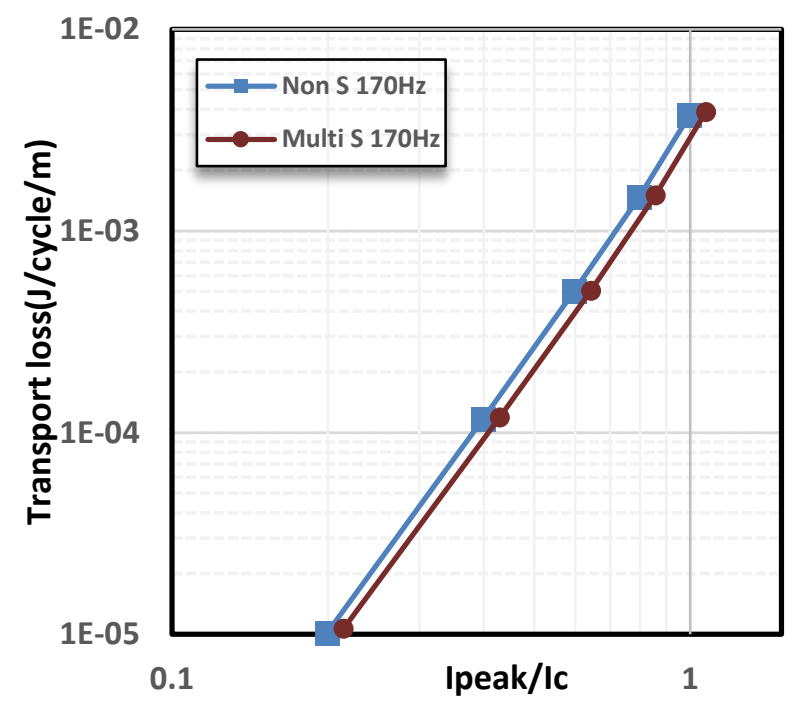

Fig. 6. Comparison of the transport AC loss with normalized current of multifilament and non-striated coils derived from simulation in $170 \mathrm{~Hz}$.

For AC losses comparison with applied current and normalized current, only two groups of results are used $(120 \mathrm{~Hz}$ and $220 \mathrm{~Hz})$. There is not a big difference between multi-filament and non-striated coils in the experimental results shown in fig.5 (a) with the same applied current. However, one can still see the transport AC losses of the multi-filament coil are slightly lower than those of the nonstriated one. The comparison results of transport AC loss plotted against the normalized current (divided by $I_{c}$ ) is shown in figure 5 (b). It is more obvious to see the difference between the two measured coils. The reduction for the multifilament coil is about $20 \%$ for the same normalized current, which is a very good feature.

According to the results in [3-8], larger width of grooves or more number of filaments for the same width of the tape leads to lower AC losses. In our experiment, there are only 4 filaments in one tape. The groove of the multi-filament tape used in our experiment is $0.03 \mathrm{~mm}$ wide. The coupling loss originating from the metallic connections among the filaments increases the total AC losses in the multi-filament coils which has been proved in [1].

\section{Simulation Results}

Fig. 6 presents the simulated results of the multi-filament coil derived from COMSOL using finite element methods with $\mathrm{H}$ Formulation $[12,15]$. We used equation 2 to calculate transport AC loss Q (J/cycle) in the simulation work [19].

$$
Q=\int_{t}\left[\sum_{1}^{N} 2 \pi \mathrm{r} \int_{S} E_{\varnothing} \cdot J_{\varnothing} d S\right] d t
$$

where ' $S$ ' is the cross section of a single turn, and ' $N$ ' denotes the total turns. $E_{\varnothing}$ and $J_{\emptyset}$ are electric field and current density in the direction of $\varnothing$. ' $r$ ' represents the radius of the Nth turn. The $J c$ we used in this simulation is constant. In figure 6, we compare the simulated results in the frequency of $170 \mathrm{~Hz}$. For both coils we used 10 turns. To compare it with the measured results we divide the transport AC loss $Q$ by the total length of the coil. As can be seen from the figure, the multi-filament coil has the lower transport AC losses as we applied the same normalized current. The AC loss reduction reaches $25 \%$. Compared with the measurement results, the simulation results show a better transport AC loss reduction. For each coil, the simulated results are lower than the measurement results. One main reason is that the model ignores the coupling losing of the coated conductor in the simulation.

\section{CONCLUSION}

In this work experimental results of AC transport loss for both multi-filament and non-striated coil wounded by YBCO coated conductors were analyzed. The critical current of multifilament coil is $66 \mathrm{~A}$ which is $5 \mathrm{~A}$ lower than the non-striated coil but with only $0.03 \mathrm{~mm}$ cut groove. Striation process indeed decreases the AC transport current but not as much as we expected. The normalization has good effects on the transport AC loss reduction in our measurement. A $20 \%$ drop can be reached for a 9.5-turn multi-filament coil with the same normalized current from the measured results. The same situation can also be found in the simulation results with a $25 \%$ decrease in the transport AC loss.

\section{ACKNOWLEDGMENT}

This study is supported by Royal Academy of Engineering Research Fellowship and EPSRC council grant (EP/P002277/1) for Dr Min Zhang. Jay Patel would like to acknowledge the ASC financial support. We also thank Superpower for kindly providing the multi-filament tape.

\section{REFERENCES}

E. Demencik, F. Grilli, A. Kario, R. Nast, A. Jung, M. Vojenciak, et al., "AC magnetization loss and transverse resistivity of striated YBCO coated conductors," IEEE Transactions on Applied Superconductivity, vol. 25, 2015.

[2] E. H. Brandt and M. Indenbom, "Type-II-superconductor strip with current in a perpendicular magnetic field," Physical Review, B: Condensed Matter, vol. 48, 1993.

[3] M. Vojenčiak, A. Kario, B. Ringsdorf, R. Nast, D. C. van der Laan, J. Scheiter, et al., "Magnetization ac loss reduction in HTS CORC®cables made of striated coated conductors," Superconductor Science and Technology, vol. 28, p. 104006, 2015.

[4] R. Nast, M. Vojeniak, E. Demencik, A. Kario, B. Ringsdorf, A. Jung, et al., "Influence of laser striations on the properties of coated conductors," Journal of Physics: Conference Series, vol. 507, p. 022023, 2014.

[5] E. Demencik, M. Vojenciak, A. Kario, R. Nast, A. Jung, W. Goldacker, et al., "AC Loss and Coupling Currents in YBCO Coated Conductors With Varying Number of Filaments," Applied Superconductivity, IEEE Transactions on, vol. 24, pp. 1-8, 2014.

[6] J. Šouc, F. Gömöry, J. Kováč, R. Nast, A. Jung, M. Vojenčiak, et al., "Low ac loss cable produced from transposed striated cc tapes," Superconductor Science and Technology, vol. 26, p. 075020, 2013.

[7] G. A. Levin, J. Murphy, T. J. Haugan, J. Scouc, J. Kovac, and P. Kovac, "AC Losses of Copper Stabilized Multifilament YBCO Coated Conductors," Applied Superconductivity, IEEE Transactions on, vol. 23, pp. 6600604-6600604, 2013.

[8] O. Tsukamoto and M. Ciszek, "Ac magnetization losses in striated ybco-123/hastelloy coated conductors," Superconductor Science and Technology, vol. 20, pp. 974-979, 2007.

[9] M. Majoros, B. A. Glowacki, A. M. Campbell, G. A. Levin, P. N. Barnes, and M. Polak, "AC losses in striated YBCO coated conductors," Applied Superconductivity, IEEE Transactions on, vol. 15, pp. 2819-2822, 2005. 
[10] M. Majoros, "Transport ac losses in striated ybco coated conductors," Journal of Physics: Conference Series, vol. 43, pp. 564-567, 2006.

[11] F. Grilli and P. A. Stephen, "Measuring transport AC losses in YBCO-coated conductor coils," Superconductor Science and Technology, vol. 20, p. 794, 2007.

[12] Z. Hong, Q. Jiang, R. Pei, A. M. Campbell, and T. A. Coombs, "A numerical method to estimate AC loss in superconducting coated conductors by finite element modelling," Superconductor Science and Technology, vol. 20, p. 331, 2007.

[13] E. Pardo, "Modeling of coated conductor pancake coils with a large number of turns," Superconductor Science and Technology, vol. 21, p. 065014, 2008.

[14] J. Šouc, E. Pardo, M. Vojenčiak, and F. Gömöry, "Theoretical and experimental study of AC loss in high temperature superconductor single pancake coils," Superconductor Science and Technology, vol. 22, p. 015006, 2009.

[15] M. Zhang, J.-H. Kim, S. Pamidi, M. Chudy, W. Yuan, and T. A. Coombs, "Study of second generation, high-temperature superconducting coils: Determination of critical current," Journal of Applied Physics, vol. 111, 2012.

[16] M. Min Zhang, J. Chudy, S. V. Wei Wang, T. A. Yiran Chen, T. A. Zhen Huang, T. A. Zhaoyang Zhong, et al., "AC Loss Estimation of HTS Armature Windings for Electric Machines," Applied Superconductivity, IEEE Transactions on, vol. 23, pp. 5900604-5900604, 2013.

[17] M. Zhang, F. Eastham, and W. Yuan, "Design and Modeling of 2G HTS Armature Winding for Electric Aircraft Propulsion Applications," Applied Superconductivity, IEEE Transactions on, vol. 26, pp. 1-5, 2016.

[18] Z. Jiahui, Z. Zhenyu, Z. Huiming, Z. Min, Q. Ming, and Y. Weijia, "Electric Measurement of the Critical Current, AC Loss, and Current Distribution of a Prototype HTS Cable," Applied Superconductivity, IEEE Transactions on, vol. 24, pp. 1-4, 2014.

[19] M. Zhang, J. Kvitkovic, J.-H. Kim, C. H. Kim, S. V. Pamidi, and T. A. Coombs, "Alternating current loss of second-generation high-temperature superconducting coils with magnetic and nonmagnetic substrate," Applied Physics Letters, vol. 101, p. 102602, 2012. 